\title{
A influência da família na autonomia e participação do cuidado do sujeito diagnosticado com Diabetes mellitus
}

The influence of the family on autonomy and participation in the care of the subject diagnosed with

\author{
Diabetes mellitus
}

\author{
La influencia de la familia sobre la autonomía y la participación em el cuidado del sujeto \\ diagnóstico con Diabetes mellitus
}

Yara Ribeiro Santos de Souza ORCID: https://orcid.org/0000-0001-7270-2202 Universidade Federal do Rio Grande do Norte, Brasil E-mail: yararibeiro001@ hotmail.com José Adailton da Silva ORCID: https://orcid.org/0000-0002-6037-7649 Universidade Federal do Rio Grande do Norte, Brasil E-mail: silva.adailton@ufrn.edu.br

Ilisdayne Thallita Soares da Silva ORCID: https://orcid.org/0000-0003-2421-8090 Universidade Federal do Rio Grande do Norte, Brasil E-mail: ilisdayne@yahoo.com.br

Talita Araújo de Souza

ORCID: https://orcid.org/0000-0002-3138-6626 Universidade Federal do Rio Grande do Norte, Brasil E-mail: talitaaraujo23@hotmail.com

Marília Rute de Souto Medeiros

ORCID: https://orcid.org/0000-0003-1817-6859 Universidade Federal do Rio Grande do Norte, Brasil E-mail: mariliarute@hotmail.com

\begin{abstract}
Resumo
O diagnóstico da Diabetes mellitus exige participação no tratamento, e mudanças na vida de quem com ela convive, e afeta não apenas o sujeito, mas também sua família. Este estudo teve como objetivo compreender a influência da família na autonomia e participação do cuidado do sujeito diagnosticado com diabetes mellitus tipo 2 . Trata-se de uma pesquisa de campo do tipo participativa, sustentada na teoria da reflexividade e com abordagem qualitativa. Dezesseis sujeitos que convivem com a diabetes participaram de encontros de um grupo de promoção da saúde. Estes participaram de oito encontros para a produção de dados, em um grupo do tipo fechado, entre os meses de maio e agosto do ano de 2017. Os resultados obtidos mostraram que os fatores que influenciam na adesão ao tratamento são multifatoriais e que nem sempre estão diretamente associados à síndrome, mas também à maneira como as pessoas vivem e se relacionam com os outros. Entre as conclusões do estudo, o apoio da família da pessoa que convive com a síndrome é essencial nesse processo, contudo, constatou-se que os participantes identificaram aspectos negativos no zelo que os familiares assumem em relação ao tratamento. Desse modo, o grupo proporcionou reflexões acerca do autocuidado dos indivíduos e da importância destes se tornarem responsáveis pelo tratamento e pela busca da autonomia para seus estilos de vida.
\end{abstract}

Palavras-chave: Diabetes mellitus; Autonomia pessoal; Relações familiares; Processos grupais.

\begin{abstract}
The diagnosis of diabetes mellitus requires participation in the treatment, and changes in the lives of those who live with it, and affects not only the subject, but also his family. This study aimed to understand the influence of the family on the autonomy and participation of the care of the subject diagnosed with type 2 diabetes mellitus. This is a participatory field research, supported by the theory of reflexivity and with a qualitative approach. Sixteen subject who live with diabetes participated in meetings of a health promotion group. These participated in eight meetings for the production of data, in a closed type group, between the months of May and August of the year 2017. The results obtained showed that the factors that influence adherence to treatment are multifactorial and that they are not always directly associated with the syndrome, but also with the way people live and relate to others. Among the conclusions of the study, the support of the family of the person living with the syndrome is essential in this process, however, it was found that the participants identified negative aspects in the zeal that the family members assume in relation to
\end{abstract}


the treatment. In this way, the group provided reflections on the self-care of individuals and the importance of them becoming resposible for the treatment and the search for antonomy for their lifestyles.

Keywords: Diabetes mellitus; Personal autonomy; Family relationships; Group processes.

\section{Resumen}

El diagnóstico de diabetes mellitus require participación en el tratamiento, y cambios en la vida de quienes conviven con ella, y afecta no solo al sujeto, sino también a su familia. Este studio tuvo como objetivo compreder la influencia de la familia en la autonomía y participación del cuidado del sujeto diagnosticado con diabetes mellitus tipo 2. Se trata de una investigación de campo participativa, sustentada en la teoría de la reflexividad y con un enfoque cualitativo. Dieciséis sujetos que viven con diabetes participaron en reunions de un grupo de promoción de la salud. Estos participaron en ocho reuniones para la producción de datos, en un grupo de tipo cerrado, entre los meses de mayo y agosto del año 2017. Los resultados obtenidos mostraron que los factores que influyen en la adherencia al tratamiento son multifactoriales y que no siempre son directamente asociado con el síndrome, pero también con la forma en que las personas viven y se relacionan con los demás. Entre las conclusions del estudio, el apoyo de la familiade la persona que vive con el síndrome es fundamental en este proceso, sin embargo, se encontró que los participantes identificaron aspectos negativos en el celo que asumen los familiares en relación tratamiento. De esta manera, el grupo brindó reflexiones sobre el autocuidado de las personas y la importancia de que se hagan responsables del tratamiento y la búsqueda de la autonomía para sus estilos de vida.

Palabras clave: Diabetes mellitus; Autonomía personal; Relaciones familiares; Procesos grupales.

\section{Introduçãa}

A Diabetes Mellitus (DM) é uma doença crônica não transmissível (DCNT), que atualmente caracteriza-se como uma epidemia e um grave desafio para a saúde pública mundial. Portanto, a alimentação inadequada, sedentarismo, obesidade dentre outros fatores fisiopatológicos estão associados ao aumento da incidência desta comorbidade (World Health Organization- WHO, 2018).

A diabetes trata-se de uma síndrome metabólica caracterizada por hiperglicemia persistente, decorrente de deficiência na produção de insulina ou na sua ação que acomete o indivíduo de qualquer faixa etária (American diabetes association, 2019). O mais comum é o diabetes tipo 2 (DM2), geralmente em adultos e idosos, ocorre quando o corpo se torna resistente à insulina ou não produz insulina suficiente. Já a diabetes tipo 1 é comum em crianças e jovens, dependente de insulina, sendo uma condição crônica na qual o pâncreas produz pouca ou nenhuma insulina por si só (ADA, 2019).

É considerada um problema mundial, e estima-se que 9,3\% dos adultos entre 20 e 79 anos - 463 milhões de pessoas vivem com a diabetes. Espera-se que haverá 578 milhões de adultos com diabetes até 2030 e 700 milhões em 2045. O Brasil ocupa o quarto lugar no mundo em número de casos, com 14,3 milhões de pessoas diabéticas (WHO, 2018).

O diagnóstico da diabetes exige participação no tratamento e mudanças na vida de quem com ela convive, e afeta não apenas o sujeito, mas também a sua família. Esta tem uma importante função no apoio emocional, mental, afetivo e no suporte físico de cada pessoa convivendo com a diabetes, formando uma rede de apoio na qual cuida e são cuidados, sendo visualizada como um núcleo de acolhimento, cuidado e atenção a esses portadores (Cortez, 2018). Desse modo, o suporte familiar permite a compreensão das mudanças relacionadas aos hábitos, a fim de melhorar a qualidade de vida.

Para que esse processo ocorra é preciso considerar as experiências das pessoas que convivem com a diabetes e de seus familiares a respeito do conhecimento sobre a síndrome, pois, conviver com a doença e suas demandas, com perspectiva de atingir o controle reflete em tarefas que exercem influências sobre a pessoa e seus familiares, designados a se adequarem à nova realidade, com o intuito de gerenciar cuidados relacionados à doença (Côrrea, 2017).

Além disso, a implantação de programas educacionais e de promoção da saúde permite aos sujeitos a aquisição de conhecimento sobre sua doença, desenvolvendo infraestrutura de apoio humano e técnico, possibilitando-os uma vida mais saudável. A falta de conhecimento resulta em uma execução deficiente das ações preventivas. Por isso se faz necessário que os profissionais conheçam a realidade de cada pessoa a fim de planejar intervenções de forma adequada que facilitem a com- 
preensão das informações oferecidas (Colombo, 2016). Intervenções educativas são essenciais nesse contexto de atendimento, com vistas à inclusão das pessoas no cuidado da própria saúde.

Destaca-se, nesse sentido, os Grupos de Promoção da Saúde (GEPS), é entendida como uma possibilidade de empoderamento a partir do referencial de cada sujeito, levando em consideração seus valores, crenças, hábitos e experiências de vida, auxiliam na construção de um referencial comum (SILVA, 2017). Sobre a autonomia caracterizam-se como a habilidade de autodeterminação, de ser independente, isto é, o indivíduo tem o poder e a habilidade de decidir ou agir sobre si próprio (Orem, 2005).

Dessa forma, o objetivo deste estudo é compreender a influência da família na autonomia e participação do cuidado do sujeito diagnosticado com diabetes mellitus tipo 2. Justifica-se, uma vez por levar em consideração a influência da família na pessoa que convive com a Diabetes Mellitus.

\section{Metodologia}

Trata-se de uma pesquisa de campo do tipo participativa apoiada no método da reflexividade, e com abordagem qualitativa. Este estudo faz parte de uma pesquisa maior, resultado de uma tese de doutorado, intitulada "Grupo Estratégico de Promoção da Saúde (GEPS): uma pesquisa participativa sobre a autonomia de pessoas vivendo com diabetes", que tem por objetivo formar estratégias de promoção à saúde que estimulem a produção da autonomia pessoal nos modos de cuidado com a saúde e com a diabetes, por meio da implantação do GEPS.

Segundo (Minayo, 2016) o estudo de abordagem qualitativa permite a compreensão de vários significados e valores adquiridos das relações existentes nas diversas situações do cotidiano. Portanto, a abordagem qualitativa é justificada, uma vez que há a preocupação em investigar e interpretar aspectos mais profundos, a fim de descrever a complexidade do comportamento humano, acerca de análises mais detalhadas sobre a investigação em questão (Minayo, 2016).

A pesquisa participativa se adequa ao estudo proposto por ser uma potente estratégia de mudança social, que colabora para que os sujeitos trabalhem em tal modificação, além de estimular a emancipação dos sujeitos. $\mathrm{O}$ tipo de pesquisa justificase, ainda, por levar em consideração a produção do conhecimento compartilhado, onde os sujeitos que convivem com a diabetes são colaboradores do estudo (Gonçalves, 2017).

A reflexividade permite que o autor não seja neutro, mas parte do processo de transformação social (Favoreto \& Cabral, 2015).

Todo o estudo foi realizado com base nos princípios da ética e bioética, estabelecidos pela resolução 466/12. Para tanto, foi submetido à análise do Comitê de Ética em Pesquisa do Hospital Universitário Onofre Lopes (HUOL), da Universidade Federal do Rio Grande do Norte (UFRN), e aprovado sob Parecer $\mathrm{N}^{\circ} 1.868 .237$ e CAAE $\mathrm{N}^{\circ}$ 61947616.4.0000.5292.

O estudo ocorreu em uma Unidade de Saúde da Família no município de Santa Cruz, interior do Estado do Rio Grande do Norte, contando com os pesquisadores da Universidade Federal do Rio Grande do Norte (UFRN) vinculados à Faculdade de Ciências da Saúde do Trairi (FACISA). O município de Santa Cruz fica localizado na Região do Trairi Potiguar e possui cerca de 39.674 habitantes distribuídos em uma área territorial de $624.356 \mathrm{~km}^{2}$ (IBGE, 2020). Como também, possui o Instituto Federal do Rio Grande do Norte (IFRN) e outras instituições que realizam trabalhos na área da educação em saúde.

Os participantes desta pesquisa são pessoas diagnosticadas com Diabetes Mellitus, cujo cuidado está sob coordenação da Equipe de Saúde da Família da qual os indivíduos estão vinculados.

$\mathrm{Na}$ seleção dos participantes para esta pesquisa, foi adotado como critério de exclusão ser menor de idade. Por sua vez, os critérios de inclusão foram: a) Ter diagnóstico de diabetes tipo 2; b) Ser cadastrado e acompanhado pela Unidade de Saúde da Família; c) Possuir prontuário familiar na Unidade; d) Não possuir nenhum tipo de distúrbio cognitivo grave que 
impossibilitasse a comunicação ou lucidez; e) Não possuir nenhum distúrbio físico que impossibilitasse a locomoção até o local de encontro dos grupos.

$\mathrm{Na}$ pesquisa foram convidados 54 participantes que residiam no território da unidade estudada, convidando-os para um encontro no dia 18 de abril de 2017 pelos Agentes Comunitários da Saúde (ACS) para participar dos encontros do GEPS, no qual estiveram presentes dezesseis sujeitos que vivem com a diabetes no decorrer dos encontros.

Para manter a privacidade do estudo, cada participante foi identificado pela letra $\mathrm{P}$ que significa as falas dos participantes, seguida do número das entrevistas, ou seja: P1, P2, P3, P4, P5, P6, P7, P8 e P9.

Estes participaram de oito encontros em um grupo do tipo fechado, entre os meses de maio e agosto do ano de 2017, onde colocou-se em prática o método denominado Grupo Estratégico de Promoção da Saúde (Silva, 2017), o qual favorece a captação das histórias de vida e as diversas experiências dos indivíduos em como lidar com a diabetes. Posteriormente, outros três encontros foram realizados para ampliar a participação dos sujeitos nas narrativas construídas no grupo e validar os dados.

Os encontros tinham como base temas que eram elencados pelos próprios participantes e sistematizados no estudo de (Silva, 2017). Os primeiros temas foram sobre a doença, como também compartilhar experiências vividas antes e depois do diagnóstico, os subsequentes eram escolhidos pelos participantes e profissionais e encerrados quando alcançado o objetivo do estudo e a saturação de falar a respeito.

Cada um dos encontros do GEPS teve um roteiro prévio elaborado após cada encontro, de modo que um encontro encadeou a necessidade do encontro seguinte.

Para a produção de dados foi utilizado um gravador de voz, onde os áudios foram transcritos fielmente em sua versão original. Estes foram organizados em narrativas que eram lidos e recebiam novas contribuições. Os dados foram analisados quanto ao conteúdo, que foram organizados em eixos temáticos, que levaram a construção das narrativas e foram validadas em três novos encontros, sob a perspectiva de (Minayo, 2016).

Além disso, foram utilizados os critérios consolidados para relatos de pesquisa qualitativa (COREQ). Os critérios incluídos no checklist podem ajudar os pesquisadores a relatar aspectos importantes da equipe de pesquisa, métodos e contexto do estudo, resultados, análise e interpretações (Tong, Sainsbury \& Craig, 2007).

A partir dos eixos temáticos que se relacionavam à abordagem da família, emergiram para esse estudo as seguintes categorias: A diabetes enquanto "herança" da família; Dificuldades para se adaptarem ao novo estilo de vida; A necessidade do apoio da família e a Repressão familiar como dificultador do processo da Diabetes Mellitus.

\section{Resultados e Discussão}

A partir da construção das narrativas foram elaboradas quatro categorias: A diabetes enquanto "herança" da família; Dificuldades para se adaptarem aos novos hábitos de vida; A necessidade do apoio da família e a Repressão familiar como dificultador do processo da Diabetes mellitus. Os resultados serão apresentados a seguir, juntamente com as falas que darão sustentação para as discussões.

\subsection{A diabetes enquanto "herança" da família}

Durante os encontros do GEPS, os relatos referentes à experiência do diagnóstico do DM foram acompanhados da rememoração dos sentimentos vivenciados naquele período, gerando confiança ao grupo. As falas seguintes são representativas dos diferentes sentimentos compartilhados no grupo acerca desse marcante momento na vida de cada participante:

P1: "Na minha família, quase todos são diabéticos". 
P2: "Eu sei que a diabetes causa muita coisa: cegueira, dormência nas pernas, tudo que é ruim acontece. Meu irmão morreu de diabetes, sem as duas pernas. Tenho medo, pois eu lembro demais quando eu fico com a diabetes mais alta".

\section{P3: "Eu sou de família diabética, meus irmãos todos! Já morreram outros; Eu já estava preparada".}

As falas apontam diversas reações frente ao diagnóstico. Apresentaram diferentes emoções como tristeza, perdas e medo, levando a reações de contestação, respectivamente. Situação semelhante ocorre com o indivíduo que reconhece as complicações da DM2, mas não o realiza o autocuidado como mostra a fala $P 6$.

Contudo, o maior problema relacionado ao descontrole da doença está em não reconhecer a condição crônica e a necessidade de procurar formas de integrá-la ao seu dia a dia. Nesse sentido, destaca-se a importância de se considerar no planejamento do cuidado, as características pessoais e as expectativas gerais em relação ao controle glicêmico, a fim de qualificar os resultados das intervenções propostas pelos profissionais de saúde (Colombo, 2016).

Do mesmo modo, os resultados desta pesquisa mostraram que os fatores que influenciam na adesão ao tratamento são multifatoriais e que nem sempre estão diretamente associados à síndrome, mas também à maneira como as pessoas vivem e se relacionam com os outros. Expressam que há diferentes sentimentos envolvidos, tais como condições de vida, contexto familiar, que nem sempre são considerados pelos profissionais ao indicarem a necessidade das pessoas realizarem os cuidados e tratamento. Entre os fatores que influenciaram favoravelmente e desfavoravelmente no tratamento, destaca-se o apoio social e familiar, a possibilidade de complicações do DM e o autocuidado.

Outros não se preocuparam com o diagnóstico DM por não ter histórico na família, tal como descrito a seguir:

\section{P4: "Na minha família não tem. Aí, eu nunca me importei. Era desligado!".}

A DM é uma doença crônica que causa complicações muitas vezes irreversíveis para o indivíduo. Portanto, o conhecimento sobre a doença é imprescindível no que se refere à precaução de complicações e equilíbrio metabólico (Sociedade Brasileira de Diabetes- SBD, 2019).

Assim, o acesso aos serviços de saúde possibilita o acompanhamento sistemático de casos, a prevenção das complicações e a utilização dos profissionais de saúde. É fundamental que a infraestrutura dos serviços, o processo de trabalho dos profissionais e a oferta diagnóstica e terapêutica respondam de forma efetiva às necessidades de saúde da população adscrita e estejam articulados com os demais pontos da rede (Colombo, 2016).

O gerenciamento de uma doença crônica, tal como o DM2, requer uma série de comportamentos de autocuidado diretamente influenciados. Por fatores intrínsecos e extrínsecos, por exemplo, a maneira com que o indivíduo reconhece e significa a doença em sua vida e o ambiente em que está inserido (SBD, 2017). Esse reconhecimento não denota apenas que o indivíduo aceite que tem DM2, mas também o integrando sua rotina, ao ambiente familiar e fora dele.

A possibilidade dos participantes de narrar a sua própria situação em que convive, pode contribuir para novas interpretações sobre o diagnóstico do DM. Quando traçamos um paralelo entre as diferentes reações dos participantes frente ao diagnóstico do DM e a necessidade de que todos, cada qual com suas especificidades envolvam-se no tratamento, percebemos o desafio que é colocado para os serviços de saúde.

\subsection{Dificuldades para se adaptarem aos novos hábitos de vida}

Nesta categoria, após o diagnóstico da DM surgem novas tarefas que afetam os familiares, a adaptação que a família irá realizar vai depender dos meios que possuem na busca pelo controle da DM. 
No entanto, a falta de apoio pela incompreensão do significado de viver com DM mostrou que, muitas vezes, a pessoa se sentia sozinha e seu esforço para o controle do DM provocava à sensação de solidão. As pessoas não compreendiam a fome e que tinham o desejo de comer coisas que lhe são proibidas. Como mostra a fala a seguir:

P5: "Eu compro doce para "o velho" toda semana... Duas latas de doce. Na hora que ele vai comer, faz questão de perguntar: quer um pedacinho? Aí eu saio, porque ele sabe que eu não como. Às vezes, me dá vontade (de comer), mas se eu pegar uma lata de doce dessas, eu como todinha".

P6: "É dificil eu comer uma coisa doce. Eu até evito comprar. Mas, meu menino (filho) é "meio safado", gosta de comprar para ele e coloca na geladeira. Têm vezes que me dá uma vontade tão grande, que eu vou e tiro um risquinho de nada e como".

É evidente a angústia dos participantes quando se referem à dificuldade de adaptação frente às necessidades impostas pela DM. Estudos também indicam a alimentação como um desafio relevante para as pessoas que convivem com o DM, sendo apontadas dificuldades significativas de seguir as orientações dos profissionais (Oliveira, Souza \& Zanetti, 2017).

As pessoas que convivem com a diabetes sabem que a eficácia do tratamento depende em parte delas, porém elas vivenciam dificuldades para se adaptarem aos novos hábitos de vida, principalmente quando se refere à alimentação, como mostra a fala P5.

As limitações e proibições impostas pela doença e seu tratamento tiram a liberdade de fazer o que se tem vontade, o que provoca um viver triste (Martins \& Rodrigues, 2019). As restrições alimentares são a principal dificuldade encontrada pelos diabéticos, pois, estas estão presentes em todos os momentos da vida dos sujeitos e representam um valor amplo na sociedade em que se vive, onde o alimento possui um valor simbólico e está vinculado à sensação de prazer, satisfação e confraternização (Pace, Ochoa \& Fernandes, 2017).

Observou-se, em alguns depoimentos, o reconhecimento de sua responsabilidade para com o próprio cuidado, como mostra a fala $P 6$. O reconhecimento do indivíduo é de sua responsabilidade frente às ações de autocuidado e um fator essencial no controle da doença e na melhora da qualidade de vida. Demonstrou ainda que a motivação elevada esteja relacionada a mudanças efetivas no estilo de vida (Orem, 2005).

Portanto, faz-se necessário que o indivíduo reconheça que, apesar de, às vezes, a implementação de mudanças em seu cotidiano ser um processo difícil, é importante, pois elas serão benéficas à sua saúde e qualidade de vida. Entretanto, a decisão pessoal constitui fator central para a realização das ações de autocuidado (Orem, 2005).

O conhecimento sobre a doença, mesmo sendo fator primordial para o bom convívio do indivíduo com DM2, por si só, não constitui fator suficiente para determinar a adoção de comportamentos positivos de autocuidado (Orem, 2005). Observa-se, na fala de P5, que embora ciente das mudanças necessárias em seus hábitos de vida para o controle adequado da doença, estas não são incorporadas em seu dia a dia. Embora fatores fisiológicos possam contribuir para essa complicação, o reconhecimento das dificuldades na manutenção das ações necessárias para o controle da doença constitui-se em fator influente na vivência dessa condição pelos indivíduos.

O autocontrole diante de certos desejos é outro desafio a ser enfrentado diariamente, este relacionado a alimentos consumidos anteriormente ao diagnóstico, que passam a ser, muitas vezes, restringidos da dieta. Acaba gerando um desconforto e é necessário o entendimento da alimentação adequada e mudanças de hábitos de vida para o convívio com a doença. É importante promover a autonomia do indivíduo porque a sua ação influencia diretamente no seu processo de saúde doença (Rezende, Dinah \& Silva, 2017). 
Muitas vezes o impacto emocional exerce uma forte influência para o desencadeamento das complicações do Diabetes Mellitus. Assim, a repressão ou a fuga da emoção pode ser causa de múltiplas doenças psicossomáticas (Federação Internacional de Diabetes- IDF, 2019). Na maioria das narrativas o diagnóstico está relacionado ao surgimento de sintomas após momentos conturbados da vida dos sujeitos, confirmando a existência de uma estreita relação entre o diabetes e a emoção, a exemplo do participante.

\subsection{A necessidade do apoio da família}

Aprendemos sobre família ao fazermos parte de uma, porém esta baseia-se na nossa própria experiência, tendo muitas vezes uma única visão da família. Apesar destas alterações, e da diversidade estrutural das famílias, continua a ser nela que a pessoa recebe suporte nas situações de crise (Nascimento \& Guedes, 2017).

A família tem um papel fundamental no enfrentamento da doença, colocando-se a serviço do bem-estar da pessoa. A DM requer mudanças nos hábitos de vida, o que repercute não apenas a vida dos sujeitos que convive com a diabetes como também a dos seus familiares. É importante reconhecer que essas mudanças geram sentimentos de preocupação nos familiares com o desejo de conviver de uma forma melhor com a DM (Nascimento \& Guedes, 2017).

A diferença entre os hábitos adotados pelos familiares e o da pessoa que convive com a DM foi um fator marcado como dificultador da reeducação alimentar, como indica a fala seguinte:

P1: "A família também precisa ajudar. A familia precisa entender o que é uma pessoa diabética em casa, não é? Eles fazerem por onde ajudar àquela pessoa. Abrir uma geladeira e estar vendo aquela coisa [doces], aí vem os desejos de comer aquilo ali".

Esse relato nos indica que incorporar uma nova forma de viver, diferente da norma construída pelo indivíduo ao longo dos anos, parece ser um processo doloroso por implicar renúncias e necessidade de formular readaptações no viver.

No entanto, a falta de conhecimento que muitos familiares possuem em relação a síndrome, pode dificultar a convivência com aqueles que possuem. Conhecer as caraterísticas dos familiares faz-se importante para saber de que tipo de apoio o sujeito dispõe no domicílio e de que forma contribui ou reforça as atividades de seu autocuidado (Orem, 2005).

As pessoas têm enorme dificuldade de mudar os hábitos alimentares, e o fator crucial é que a família pode contribuir para facilitar ou dificultar este processo, e isso foi identificado nas falas dos indivíduos nos encontros do GEPS. As informações e orientações a respeito dos conhecimentos e atitudes sobre a DM podem favorecer a mudança de hábitos de vida associados à dieta e à prática de atividade física.

Conforme observado nas falas dos sujeitos com DM, é perceptível a ausência de informações e apoio. É neste cenário que as redes de apoio podem auxiliar. A presença do profissional da área da saúde nas falas dos sujeitos expressa a necessidade contínua de informação e apoio, o que gera a reflexão sobre a necessidade de se educar não somente os usuários, mas, sobretudo, seus companheiros e membros de convívio diário (Colombo, 2016).

A prática educativa quando é reflexiva, disposta ao diálogo e à resolução de problemas, se mostra passível de apresentar bons resultados, tanto nos níveis glicêmicos, quanto na autoconfiança e satisfação com o tratamento (IDF, 2019).

A atuação de companheiros, familiares e amigos próximos tem impacto positivo no tratamento. Companheiros que se dispõe a dar apoio, acompanhar as práticas de atividade física e ter uma alimentação regrada, ajudam a promover melhores resultados a partir do momento que incentivam e acompanham uma vida saudável (Pennafort, Queiroz, Nascimento \& Guedes, 2017).

É evidente a angústia dos participantes quando se referem à dificuldade de adaptação frente às necessidades impostas pela DM. Estudos também indicam a alimentação como um desafio relevante para as pessoas que convivem com o DM, sendo 
apontadas dificuldades significativas de seguir as orientações dos profissionais (Pennafort, Queiroz, Nascimento \& Guedes, 2017).

\subsection{A repressão familiar como dificultador do processo da diabetes mellitus}

Estudos apontam que quando a família não convive em um clima de harmonia e que os conflitos são recorrentes, os efeitos do DM se tornam evidentes para a pessoa que convive com a DM. O ato de cuidar é uma responsabilidade difícil, acompanhar e conviver com o adoecimento provocam reações negativas produzindo mudanças na dinâmica familiar, o que pode gerar uma desqualificação no cuidar (Côrrea, 2017).

A doença afeta a família e seus membros de forma diferenciada em intensidade e qualidade, visto que cada família atribui significado diferente a uma mesma situação de doença, o que afeta o estilo de enfrentamento de cada pessoa e da sua família.

P3: "Eu fui uma que quase morro com um pedaço de doce (...) achando que minha filha estava chegando. (...) se eu soubesse [que não era ela], eu teria comido mais".

P5: "Eu como tudo. Certa vez, eu ia morrendo engasgada. Chegou alguém, pensei que era minha filha... Como escondida, mas depois eu digo a ela".

A participação da família nos cuidados e tratamento da pessoa com DM é uma situação que nem sempre beneficia a adesão ao tratamento e a convivência harmoniosa com a doença (Côrrea, 2017). Quando a família se coloca de maneira autoritária e controladora, punindo os excessos, pode gerar revolta, tristeza, desmotivando-a para a adesão ao tratamento ou, como mostrada nas falas anteriores, levando a pessoa agir de forma incorreta, escondida e com medo.

A cumplicidade e a união entre os membros da família estão relacionadas a uma adesão de qualidade ao tratamento pelo paciente diabético, por outro lado os conflitos familiares representam barreiras a essa adesão de efetiva (Borba, 2018).

Essa situação alerta para a importância de incluir a família na educação em saúde, de forma que ela seja um importante suporte e que ajude na adesão ao tratamento, pois se as ações dos familiares não forem afetivas podem interferir nos esforços do autocuidado da pessoa com DM (Orem, 2005).

As mudanças relacionadas ao diabetes estão associadas aos padrões, comportamentos, sentimentos e a compreensão que fazem sobre a doença, isso influencia no desempenho familiar, na busca de contribuições que proporcionam novos recursos de enfrentamento. Para tanto, é importante observar e obter dados por meio de uma situação de vida real (Cortez, 2018).

Além da motivação (ou falta dela), a presença de sentimentos negativos associados ao DM sugere repressão familiar, uma condição comumente observada que pode afetar negativamente a forma como os indivíduos cuidam de si mesmos. É importante ressaltar que usuários apresentam sintomas como perda de interesse pelo seu cuidado, cansaço e baixa auto estima (Orem, 2005).

Quer seja barreiras relacionadas à alimentação, que seja atividade física, o que geralmente impede a essas pessoas de se tornarem ativos e sentirem prazer em cuidar de si são os sentimentos negativos associados à necessidade de mudar hábitos. O estresse gerado pela obrigação de seguir um padrão alimentar foi perceptível nas falas dos participantes do presente estudo, corroborando com achados na literatura de que o fato de não ser capaz de mudar o comportamento é estressante e gera sentimentos negativos na promoção do autocuidado (Cortez, 2018).

Por isso, faz-se necessário o profissional de saúde inserir a família no processo de cuidado diário e envolvê-la como parte integrante do seu cuidar, através da educação em saúde. 


\section{Conclusão}

Ao analisarmos os sentimentos gerados a partir do diagnóstico da DM, a forma como se dá a convivência com a doença no cotidiano, os fatores relacionados com a mudança de hábitos, percebem-se as principais diferenças que os participantes apresentaram, seja na forma como se colocam diante dos desafios, das estratégias desenvolvidas para lidar com a DM, seja nos apoios disponíveis no contexto familiar e nos entraves encontrados.

$\mathrm{O}$ apoio da família da pessoa que convive com a síndrome é essencial nesse processo, contudo, constatou-se que os participantes identificaram aspectos negativos no zelo que os familiares assumem em relação ao tratamento. Desse modo, o grupo proporcionou reflexões acerca do autocuidado dos indivíduos e da importância destes se tornarem responsáveis pelo tratamento e pela busca da autonomia para seus estilos de vida.

Nessa perspectiva, o grupo propiciou um clima aberto para livres expressões de ideias e sentimentos, permitindo aos participantes relatarem suas experiências, expressando seus sentimentos e buscando sua autonomia.

Por fim, destaca-se como limitação deste estudo o fato de o levantamento de informações ter sido com 16 pessoas de uma realidade de saúde similar, em um único território de saúde.

\section{Referências}

ADA. (2019). Standards of Medical Care in Diabetes - 2019. American Diabetes Association (ADA). Diabetes Care. $42(1), 62-7$.

Borba, A. K. O. T., et al. (2018). Fatores associados à adesão terapêutica em idosos diabéticos assistidos na atenção primária de saúde. Ciência. saúde colet. Rio de Janeiro, 23(3), 953-961.

Cortez, D. N., \& Reis, I. A., \& Souza, D. A. S., \& Macedo, M. M. L., \& Torres, H. C. (2018). Complicações e o tempo de diagnóstico do diabetes mellitus na atenção primária. Acta Paul Enferm. 28(3): 250-255. https://dx.oi.org/10.1590/1982-0194201500042.

Corrêa, K. et al. (2017). Qualidade de vida e características dos pacientes diabéticos. Ciência \& Saúde Colet. 22(3), 921-930. https://doi.org/10.1590/141381232017223.24452015 .

Colombo, E. (2016). Reflexividade e escrita. Educ. Rev. Educação. 41(1), 15-26.

Favoreto, C.A.O., \& Cabral, C.C. (2015). Narrativas sobre o processo saúde - doença: experiências em grupos operativos de educação em saúde. Rev. Interface Comun Saúde Educação. 13(28),7-18.

Federação Internacional de Diabetes (IDF). (2019). Atlas de Diabetes da IDF. Bélgica.

Gonçalves, N. E. X. M., \& Zanetti, M. L., \& Neiva, C. M. et al. (2017). Conhecimento de indivíduos com diabetes mellitus na estratégia de saúde da família. Rev. de Enferm. 11(7):2779-87.

Martins, M. M. F. \& Rodrigues, M. L. (2019). Diabetes: Adesão do Paciente e o Papel da Família nessa Nova Realidade. Rev. Aten. Saúde, São Caetano do Sul, 17(59), 95-102.

Minayo, M. C. S. (2016). O desafio do conhecimento: pesquisa qualitativa em Saúde, (14a ed.), Editora São Paulo.

Nascimento, L. C., \& Guedes, M. V. C. (2017). Rede e apoio social no cuidado familiar da criança com diabetes. Rev. Bras. de Enferm. 69(5): 912-9. http://dx.doi.org/10.1590/0034-7167-2015-0085.

Oliveira, N. F., \& Souza, M. C. B. M., \& Zanetti, M. L., \& Santos, M. A. (2017). Diabetes Mellitus: desafios relacionados ao autocuidado abordados em Grupo de Apoio Psicológico dados em Grupo de Apoio Psicológico. Rev. Bras. Enferm. 64(2): 301-7.

Orem, D. E. N. (2005).Concepts of practice. Mosby.

Pace, A. E., \& Ochoa, V. K., \& Caliri, M. H. L., \& Fernandes, A. P. M. (2017). O conhecimento sobre diabetes mellitus no processo de autocuidado. Rev. Latino Americ. Enferm. http://dx.doi.org/10.1590/S0104-11692006000500014.

Pennafort, V. P. S., \& Queiroz, M. V. O., \& Nascimento, L. C., \& Guedes, M. V. C. (2017). Network and social support in family care of children with diabetes. Rev. Bras. Enferm. 13(1):346-52. http://dx.doi.org/10.1590/0034-7167-2015-0085.

Silva, A. B. et al. (2017). Prevalência de diabetes mellitus e adesão medicamentosa em idosos da Estratégia Saúde da Família de Porto Alegre/RS. Cad. saúde colet. 24(3), 308-316. https://doi.org/10.1590/1414-462x201600030017.

Sociedade Brasileira de Diabetes (SBD). (2017). Diretrizes da Sociedade Brasileira de Diabetes 2017-2018.

Sociedade Brasileira de Diabetes (SBD). (2019). Conduta terapêutica no diabetes tipo 2: Algoritmo SBD 2019. 
Research, Society and Development, v. 10, n. 4, e56710414113, 2021

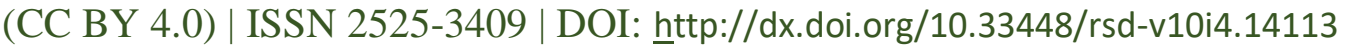

Tong, A., \& Sainsbury, P., \& Craig, J. (2007). Critérios consolidados para relatar pesquisas qualitativas (COREQ): uma lista de verificação de 32 itens para entrevistas e grupos de foco. International Journal for Quality in Health Care, 19(6), 349-357. https://doi.org/10.1093/intqhc/mzm042.

World Health Organization (WHO). (2016). Global report on diabetes.

World health organization (WHO). (2018). Noncommunicable diseases in the Region of the Americas: facts and figures. 